\title{
Estimating Quality Bounds of JPEG 2000 Compressed Leukocytes Images
}

\author{
Alexander Falcón-Ruiz ${ }^{1}$, Juan Paz-Viera ${ }^{1}$, and Hichem Sahli ${ }^{2}$ \\ ${ }^{1}$ Center for Studies on Electronics and Information Technologies, Universidad Central de \\ Las Villas, Carretera a Camajuaní km 5 ̌ㅗ, Santa Clara, VC, CP58430, Cuba \\ \{afalcon, jpaz\}@uclv.edu.cu \\ ${ }^{2}$ Vrije Universiteit Brussel, Dept. Electronics \& Informatics, VUB-ETRO, \\ B-1050 Brussels, Belgium, \\ sahli@etro.vub.ac.be
}

\begin{abstract}
Several pathologies are detected by counting different types of leukocytes indigital microscopic images. However, manipulation of these images, i.e. storage and/or transmission, can be complicated by the large sizes of the files containing them. In order to tackle this particular situation, lossy compression codecssuch as JPEG2000 have been employed while preserving the overall perceived image quality. In this paper a strategy based on objective quality metrics and performance of segmentation algorithms is proposed for the estimation of the maximal allowable compression rate $(\mathrm{CR})$ where deterioration introduced in the images by the JPEG 2000 codec does not affect identification of white blood cells. Results indicate that the estimated value lays around CR = 142:1 as measured by the metrics employed.
\end{abstract}

Keywords: JPEG 2000, microscopic images, leukocytes, compression, segmentation.

\section{Introduction}

Several pathologies such as acquired immunodeficiency syndrome,cancers, or chronic infections, are detected nowadays as a specialistobserves and extracts information from images containingwhite blood cells,also called leukocytes.Traditionally the expert select an area of interest in a peripheral blood or bone marrow slide, and by using a microscope, detectsdifferent types of leukocytes, increasing the counts for each one, providing important information to doctors in the diagnosis of such diseases.

Microscope-based biomedical imaging technique is characterized by large file sizes due to the bit depths employed and the high resolution properties of the digital acquisition devices. Some issues might arise when manipulating these images, i.e. during storage of everyday image production and/or transmission through digital communication networks [1], [2]. The amount of such images obtained in everyday practice, depending on the type of studies required for every particular detection task, can be enormous. 
Although diagnosis is not recommended over compressed images, there has been an effort to employ lossycodecsfor images that undergo second evaluation as part of a second opinion or a follow-upprocess. These algorithms are reported to have an order of magnitude higher in compression rate (CR) in comparison to losslesscodecs.

One ofthesecodecs is JPEG 2000 (ISO 15444-1), based on the wavelet transform and added to DICOM standard around November 2001, in Supplement 61: JPEG 2000 Transfer Syntaxes[3], [4].

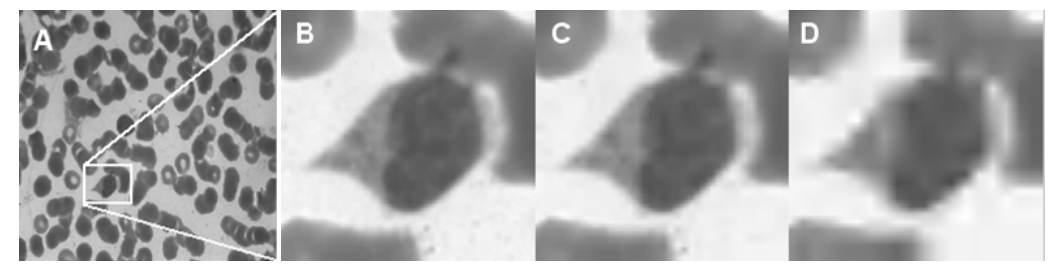

Fig. 1. Section Ashows a 1536V x 2048H pixel size bitmap image which occupies 9.00 MB of disk space. Sections B, C and D showa region containing a monocyte extracted from A after it is compressed at 3 different JPEG 2000 CRs, i.e. 50:1, 100:1 and 200:1. The edges, texture and contrast are severe distorted as compression rate increases.

Although JPEG2000 has been adopted by DICOM standard, there are still no regulations for the use of itslossymode where, the higher the CRs are, the more distortion is introduced in the image, affecting particularly edge definition and so jeopardizing the correct identification of the structures and the diagnosis made through these images [5]. The example in Fig. 1 shows a typical image and a Region of Interest (ROI) extracted from this imageafter compression at different CRs.

Several researches have been carried out in order to establish a CRlimit for specific image types where the overall perceived image quality is not perceptually affected when using lossycodec [6], [7], [8]. In this paper, we propose estimating the maximum allowable CR where deterioration introduced, by the codec, in the images does not affect the quality of leukocytes images. The estimation is based on objective quality metrics, and its performance is evaluated using several segmentation algorithms.

\section{Materials and Methods}

\subsection{The Images}

Images were acquired using a Micrometrics 318CU CMOS digital camera, resulting in 24-bit color pictures of $2048 \mathrm{H} \mathrm{x} 1536 \mathrm{~V}$ size. The camera was attached to an Accuscope 3016PL trinocular microscope with 100x oil immersion objective and 10x eyepieces. For the test, we selected 15 images per leukocyte class, where theclasses of interest were: lymphocytes, monocytes, neutrophils, basophils and eosinophils. Some manually cropped images are shown in Fig. 2. 


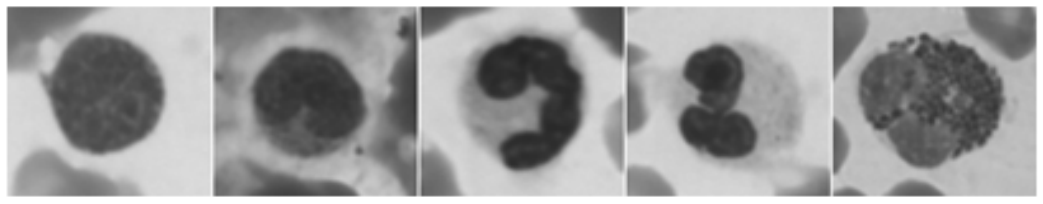

Fig. 2. Leukocytes. Left to right: lymphocyte, monocyte, neutrophil, basophil, eosinophil

Leukocytes classification is characterized by the observation, detection and classification of details or singularities within those images. A specialized observer extracts features such as shape, texture and color from them in order to classify the cells according to the types mentioned above. The lossycompression of such images might introduce distortions that directly affect the way in which those details are perceived as CR increases. Their preservation is crucial for assuring correct classification of leukocytes.

\subsection{Compression with JPEG 2000 Codec}

The implementation of JPEG2000 known as JasPer[9]was employed.Each entire image (as in Fig. 1 A) was compressed in a wide range of CR values from 33:1, where images show little degradation in quality, up to 1000:1, where image quality is highly degraded, the deterioration observed in the images is significant, cells loose important information such as edge definition, and contrast is also affected.Using a compression factor $(\mathrm{CF}=1 / \mathrm{CR})$ step of 0.001 , a set of 30 compressed images ( $\mathrm{CF}$ from 0.001 to 0.030) was produced. Later on, the ROIs were extracted from the uncompressed and every reconstructed image.

The CR is calculated as the necessary memory space (in bytes) for allocating uncompressed image divided by the number of bytes necessary for allocating the same image in its compressed format.

\subsection{Quantitative Measures}

Traditionally, the overall estimation of image quality has been carried out with the calculation of several objective uni-variate and bi-variate metrics, altogether with subjective criteria involving human observers. Their reliability in different situations and image types has been also widely investigated by many authors [1], [2], [10].

For our particular research the following bi-variate measures are chosen:

- The Peak Signal-Noise Ratio $(P S N R)$ :considering $X(i, j)$ as the uncompressed image and $Y(i, j)$ the restored one, PSNRis defined as:

$$
\operatorname{PSNR}(d B)=10 \cdot \log _{10}\left(\frac{M A X p^{2}}{M S E}\right)
$$

where $M A X p=2^{B}-1, B$ is the image bitdepth and $M S E$ (mean square error) is defined as: 


$$
M S E=\frac{1}{m \cdot n} \sum_{i=1}^{m} \sum_{j=1}^{n}(X(i, j)-Y(i, j))^{2},
$$

where $m$ and $n$ are the number of rows and columns in the image, respectively.

- The spectral distance $(S D)$ :a measure of distance between uncompressed and reconstructed Fourier domainimages given by:

$$
S D=\frac{1}{m \cdot n} \sum_{i=1}^{m} \sum_{j=1}^{n}(|\varphi(i, j)|-|\hat{\varphi}(i, j)|)^{2},
$$

where $\varphi(i, j)$ and $\hat{\varphi}(i, j)$ are the imaginary parts ofFourier transforms of uncompressed and restored images, respectively.

- The gain in Contrast to Noise ratio $(g C N R)$ is defined as:

$$
\operatorname{gCNR}(d B)=10 \cdot \log _{10}\left(\frac{C N R_{X}}{C N R_{Y}}\right)
$$

where $C N R_{X}$ and $C N R_{Y}$ are the contrast-to-noise ratios in the uncompressed and reconstructed images respectively calculated as $C N R_{i}=\left(\bar{X}_{i 2}-\bar{X}_{i 1}\right) / \sigma_{i}$, with $\bar{X}_{i 1}$ and $\bar{X}_{i 2}$ being the mean values of intensity from two different regionsin image $i$ and $\sigma_{i}$ the standard deviation of noise in same image.

- The structural similarity index (MSSIM):a powerful measure proposed by Wang et al. [10] was also employed. It can be calculated as:

$$
\operatorname{MSSIM}(X, Y)=\frac{1}{M} \sum_{i=1}^{M} \operatorname{SSIM}\left(x_{i}, y_{i}\right),
$$

whereMis the number of image blocks $x_{i}$ and $y_{i}$ of uncompressed and reconstructed image respectively and SSIM calculated as:

$$
\operatorname{SSIM}(X, Y)=\frac{\left(2 \mu_{X} \mu_{Y}+C_{1}\right)\left(2 \tau_{X Y}+C_{2}\right)}{\left(\mu_{X}^{2}+\mu_{Y}^{2}+C_{1}\right)\left(\tau_{X}^{2}+\tau_{Y}^{2}+C_{2}\right)},
$$

where $\mu_{x}$ and $\mu_{y}$ are the luminance values, $\tau_{X}$ and $\tau_{Y}$ the contrast estimation values for uncompressed and reconstructed image respectively and $\tau_{X Y}=\frac{1}{N-1} \sum_{i=1}^{N}\left(x_{i}-\right.$ $\left.\mu_{x}\right)\left(y_{i}-\mu_{y}\right)$. The constants $C_{l}$ and $C_{2}$ are placed to avoid instability: $C_{i}=\left(K_{i} L\right)^{2}$ where $L=255$, for 8bpp images and $K_{i}<<1$.

All bi-variate calculations are made between the uncompressed image and every reconstructed image after being compressed at each CR value in the interval studied.

\subsection{The Segmentation Algorithms}

Typically, leukocytes identification is based on visual inspection of individual images of wider Field Of View than the size of individual cells and containing other structures as well as noise and/or artifacts. The approach of having experts dedicated to this task is time consuming, exhausting and prone to human error, requiring frequent repetitions to validate results [11].These situations, altogether with the great amount of images necessary to achieve a diagnosis, encourage scientists to develop segmentation algorithms as an early step for automated classification. 


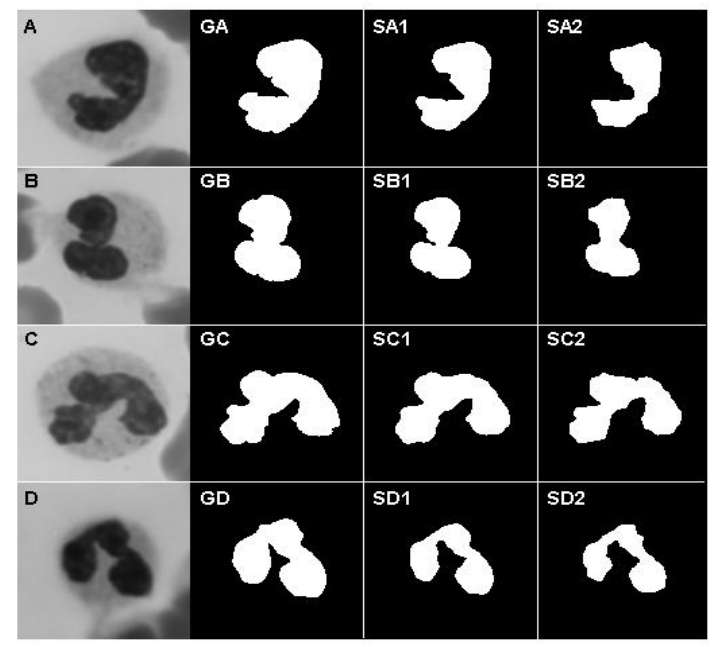

Fig. 3. Images (A-D) in first column are green component sections containing leukocytes extracted from bigger images as in Fig. 1 section A. Second column (GA - GD) contains the manually extracted Ground Truths for these sections, while columns three and four contains the segmentation results at $\mathrm{CR}=33: 1$ (SA1-SD1) and $\mathrm{CR}=1000: 1$ (SA2 - SD2).

These algorithms are conceived to analyze the images acting as secondary readers where they reanalyze the image after the initial diagnose by the physician. They are also designed to objectively quantify features in a robust, reliable and reproducible manner.

In the present paper, to assess the CR three automatic segmentation algorithms are tested over a set of leukocytes images, each one compressed at 30 different $\mathrm{CR}$ values within the interval 33:1 to 1000:1, i.e. Otsu's method [12], Active Contours (AC)method [13, 14] and the Mixture of Gaussians (MoG) method [15]. For assessing the segmentation results, of each of the proposed methods, applied at specific CR, theHausdorff distance [16], between Ground Truths (GTs) and segmentation results have been estimated.GTswere selected in each ROI at initial state, i.e. without compression. Fig. 3 shows some of the ROIs from the test images set, their GTs and segmentation results at minimum and maximum CR.

\section{Results}

Fig. 4 shows four rate-distortion curves for the four different quality metrics calculated over the ROIs within the CR interval investigated and averaged over the 15 images in the test set. From the graph, it is observed that metrics such as PSNR and $g C N R$ show a stronger dependency with CR variation while $S D$ and MSSIM show less dependence with CR.

A nick point is observed in the curves near $\mathrm{CR}=142: 1 \quad(\mathrm{CF}=0.007)$. For $\mathrm{CR}$ values bigger that this, image quality is severely distorted. At this point PSNR is around 91 


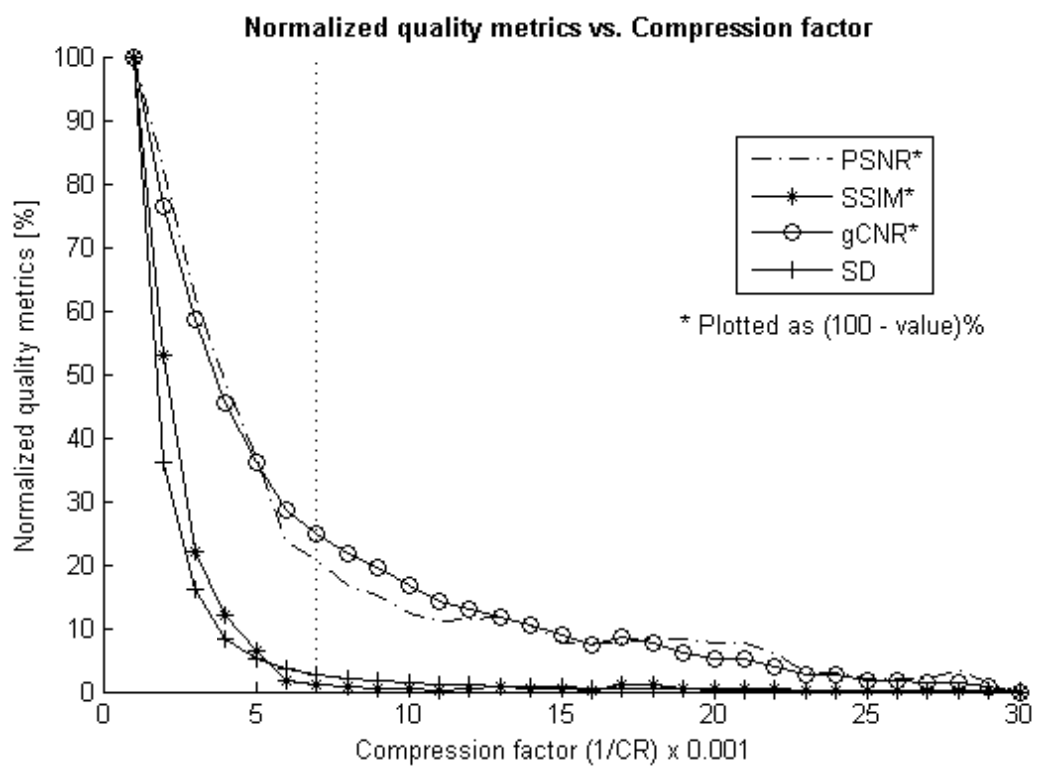

Fig. 4. The objective metrics are shown in a percent scale. Metrics such as PSNR and $g C N R$ show a stronger dependency with variation in CR while metrics such as $S D$ and MSSIM show less dependence with $\mathrm{CR}$. The nick point in the curves at $\mathrm{CR}=142: 1$ suggests a lower CR bound. For CRs bigger that this, image quality is severely distorted.

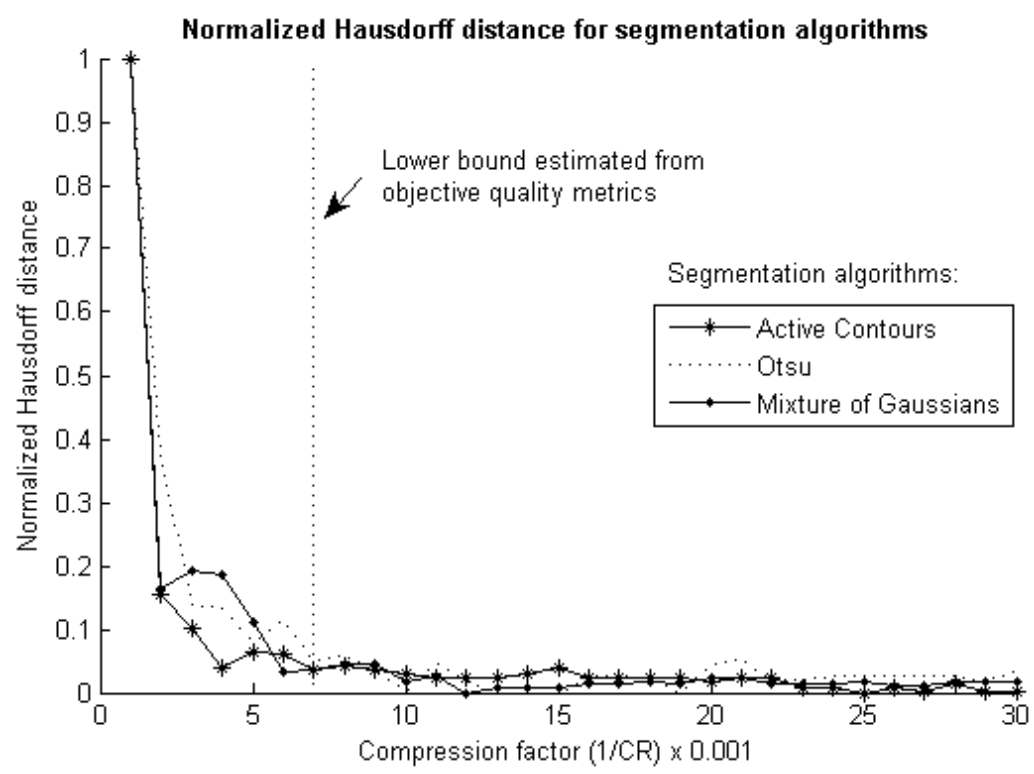

Fig. 5. Normalized Hausdorff distance for the three segmentation algorithms tested. Dotted line indicates the estimates lower bound in correspondence with previous results from objective quality metrics 
$\mathrm{dB}, g C N R$ is around20dB, SD is 1.19 units and MSSIM is 0.98 . At this CR, file size is reduced from $9 \mathrm{MB}$ to approximately $65 \mathrm{~KB}$.

Fig. 5 shows the normalized Hausdorff distances for the three segmentation algorithms tested. Although in this graph the three methods show similar behavior as quality metrics, Otsu's method had the best performance in our experiment with lower Hausdorff distance (HD) to the GT (at $\mathrm{CR}=33: 1, H D_{O t s u}=4.5, H D_{M o G}=8.2$, and $H D_{A C}=10.1$ Hausdorff distance units). The Hausdorff distance for CRs below 142:1 has a standard deviation below $5 \%$ of the Hausdorff distance for the maximum CR tested.

\section{Conclusions}

The analysis with objective metrics suggestedan interval of CR values from 33:1 up to 142:1 where is safe to use JPEG 2000. This initial and partial result is later confirmed by the automatic segmentation algorithms tested which agrees in the upper most CR value of 142:1.

Both, metrics for evaluating objective quality distortions and the performance of segmentation algorithms, are considered representative for estimating quality degradation caused by the lossy codec.

The result presented are preliminary and lack of subjective experience in interpreting this type of images. A more complex investigation including subjective evaluation should be carried out in order to precise the bounds for lossy compression. Nevertheless, a CR limit of 142:1 was estimated through both metric types as a limit for using JPEG 2000 compression in leukocytes identification tasks.

\section{Acknowledgements}

The authors would like to thank Alberto Taboada-Crispí for the analysis and review, the Canadian International DevelopmentAgency Project Tier II-394-TT02-00 and the Flemish VLIR-UOS Programme for InstitutionalUniversity Cooperation (IUC) for partly supporting this investigation.

\section{References}

1. Acharya, T., Ray, A.K.: Image processing Principles and applications. John Wiley \& Sons, Inc., Hoboken (2005)

2. Lau, C., et al.: Telemedicine.Handbook of Medical Imaging. In: Kim, Y., Horri, S. (eds.), vol. 3, pp. 305-331. SPIE, Bellingham (2000)

3. Clunie, D.A.: DICOM Supplement 61: JPEG 2000 Transfer Syntaxes (2002), ftp://medical.nema.org/medical/dicom/final/sup61_ft.pdf

4. Rabbani, M., Joshi, R.: An overview of the JPEG2000 still image compression standard. 1, Signal Processing: Image Communication 17, 3-48 (2002)

5. Foes, D.H., et al.: JPEG 2000 compression of medical imagery. In: SPIE Proc., San Diego, California, vol. 3980 (2002) 
6. Penedo, M., Lado, M.J., Tahoces, P.G., Souto, M., Vidal, J.J.: Effects of JPEG2000 data compression on an automated system for detecting clustered microcalcifications in digital mammograms. IEEE Trans. on Information Technology in Biomedicine 10(2) (2006)

7. Zhang, Y., Pham, B., Eckstein, M.P.: Evaluation of JPEG2000 encoder options: human and model observer detection of variable signals in X-Ray coronary angiograms. IEEE Trans. on Med. Imagin. 23(5) (2004)

8. Paz, J., Pérez, M., Schelkens, P., Rodríguez, J.: Impact of JPEG 2000 Compression on Lesion Detection in MR Imaging. Journal of Medical Physics 36(11), 4967-4976 (2009)

9. Adams, M., Kossentini, F.: JasPer: a software based JPEG2000 codec implementation. In: Proc. of IEEE International Conference on Image Processing, Vancouver, British Columbia, Canada. Institute of Electrical and Electronics Engineers, vol. 2, pp. 53-56 (2002)

10. Wang, Z., Bovik, A.C., Sheikh, H.R., Simoncelli, E.P.: Image Quality Assessment: FromError Visibility to Structural Similarity. IEEE Trans. on Image Proc. 13(4) (2004)

11. Lee, J.K.T.: Interpretation accuracy and pertinence. American College of Radiology 4 (2002)

12. Otsu, N.: A Threshold Selection Method from Gray-Level Histograms. IEEE Transactions on Systems, Man and Cybernetics 9(1), 62-66 (1979)

13. Kass, M., Witkin, A., Terzopoulos, D.: Snakes: Active contour models. International Journal of Computer Vision 1(4), 321-331 (1988)

14. Chan, F.C., Vese, L.A.: Active Contours Without Edges. IEEE Transactions on Image Processing 10(2), 266-277 (2001)

15. Gupta, L., Sortrakul, T.: A gaussian-mixture-based image segmentation algorithm. Pattern Recognition 31(3), 315-325 (1998)

16. Huttenlocher, D., Klanderman, G.A., Rucklidge, W.J.: Comparing Images Using the Hausdorff Distance. IEEE Transactions on Pattern Analysis and Machine Intelligence 15(9), 850-863 (1993) 